\title{
Case Study: Personalized self-hypnosis method for Irritable bowel syndrome
}

\author{
Article by Koo Kian Yong \\ Master of Science in Clinical Psychology, Texila American University, Malaysia \\ Email:newmindcentre@gmail.com
}

\subsection{Identifying Information}

Clinical hypnotherapy is proven by many research studies as an evidence-based practice. Hypnotherapy is classified as a complementary therapy which regulated by Ministry of Health in Malaysia. Irritable bowel syndrome (IBS) is a common disorder that many patients suffered in Malaysia. However, there is still lack of practitioner who specialized in IBS and offer effective intervention. According to a recent study (Lindfors el at., 2012), gut-directed hypnotherapy is one of the most effective intervention for IBS related issues and the result able to sustain up to 1 year. However, traditional gut-directed hypnotherapy involves 12 sessions of 1 hour therapy session. This case study will demonstrate the advantages of personalized self-hypnosis method which combine with a brief version of gut-directed hypnotherapy session which only involve 3 sessions. Treatment is tailored for Eric Liu, a male client who is 35 years old and diagnosed with Irritable bowel syndrome by medical doctor.

\subsection{Presenting Complains}

- Irritable bowel syndrome (IBS) related issues

- Stomach and liver area discomfort

- Diarrhea

- Nervous and anxiety issues

- Concentration issues

\subsection{History of presenting issues}

Eric Liu reports that he is having IBS problem since 5 years ago after food poisoning incident. He reports to us that medical doctor told him that he is suffering with IBS problem. He reports that he is unable to relax, fear of stomach discomfort problem, and fear of worst things will be happened on him. He always feel stomach discomfort before he ready to go out from the house or he feels anxious. Sometimes, he even went to toilet more than 3 times before he ready to go out from the house.

At work, he faces difficulty in concentration and engage in task due to IBS issues. He finds that he cannot focus and pay attention to others during the conversation. All these problems are bothering him a lot. He wants to improve his quality of life.

During the past month, there is more than 3 times a week that he feels stomach and liver area discomfort. He went to see medical doctor and traditional Chinese medicine practitioner often. However, he remarks that he can't see much improvement after received treatment from them. In recent, he feels that his symptoms become more serious. Thus, he makes a decision to try on neuro-hypnotherapy. 
South American Journal of Academic Research

Special Edition May 2016

\subsection{Behavioural observation}

Appearance:

He appears well. Dress in a relaxed clothing style

Behaviour:

Nervousness but friendly.

Willingness to listen.

Speech and language:

He can communicate well. Speech is rapid with long pause

Affect:

He was quite anxious about what was going to happen during the session

Attention, concentration and impulsivity:

He is good in listening and attention skill.

Impatient.

\subsection{Summary/recommendations}

The initial interview session indicate that he is concern about his IBS issues, anxiousness and life quality.

Based on the initial interview session, the following ideas are suggested to help to deal with his issues, namely:

i. Coping skill for anxiousness

ii. Neuro-hypnotherapy (gut directed method) for IBS issues

iii. Personalized self-hypnosis method

iv. Psychoeducation: Macronutrient Mood Therapy

\subsection{Therapy session}

\section{Neuro-hypnotherapy}

i. Session one: Hypnotic suggestibility testing and gut directed hypnotherapy

- An individualized self-hypnosis plan that is tailored according to his brainwaves and hypnotic suggestibility testing result is suggested for better therapeutic outcome. Selfhypnosis approach has to be based on his hypnotic suggestibility testing result instead of traditional self-hypnosis techniques.

- His brainwaves show that she is responsive on certain hypnotic suggestion such as gustatory system, imagination learning system and visualization etc.

- Hi-Beta fast brainwaves which are associated with stress, anxiety and tension was remaining low during the session. This could indicate that he is remaining calm during the session. However, there is a cross over in between Alpha brainwave and Hi-Beta brainwave. This could indicate that He was doing his best to pay attention and follow to the therapist's suggestion and instruction.

- Slow brainwaves such as delta and theta was remaining high during the session. The gap between Delta and Hi-Beta brainwaves are considered as big gap. However, the gap between Theta and Alpha brainwaves are not significant big enough. This could indicate that he is in a calm and relaxed mood. 


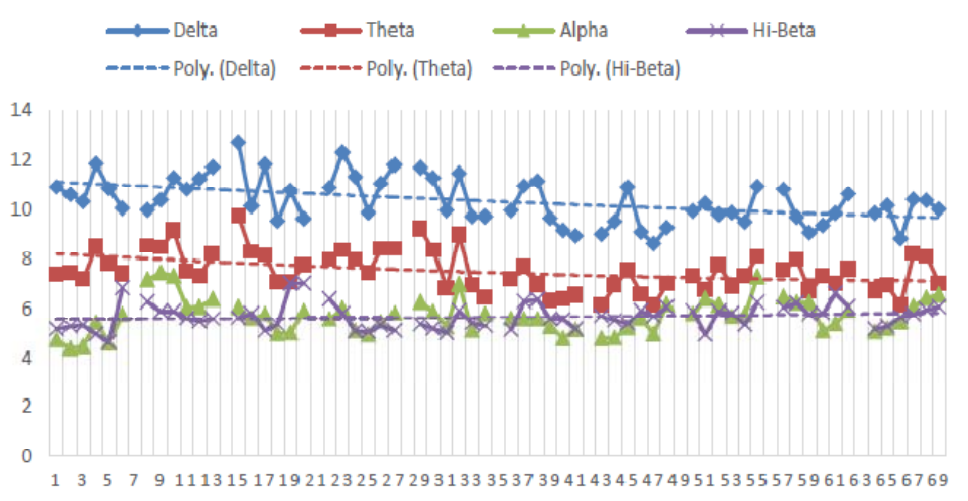

The graph above shows the session on 13th May 2015

\section{Session two: practicing personalized self-hypnosis, coping skill and gut directed hypnotherapy}

- Hi-beta fast brainwaves were higher than theta and alpha at the first 4 period. This could indicate that he was paying attention to the suggestion and his mind is actively engage in the given task.

- Slow brainwaves such as Delta, Theta and Alpha were keep increasing during the session. This could indicate that he was going into a deeper trance state.

- Another clue that he went into a deeper trance state is the gap between alpha and theta was becoming bigger during the session. From the graph, we can notice that the theta is higher than alpha. This could indicate that client was experiencing a subconscious mental activities.

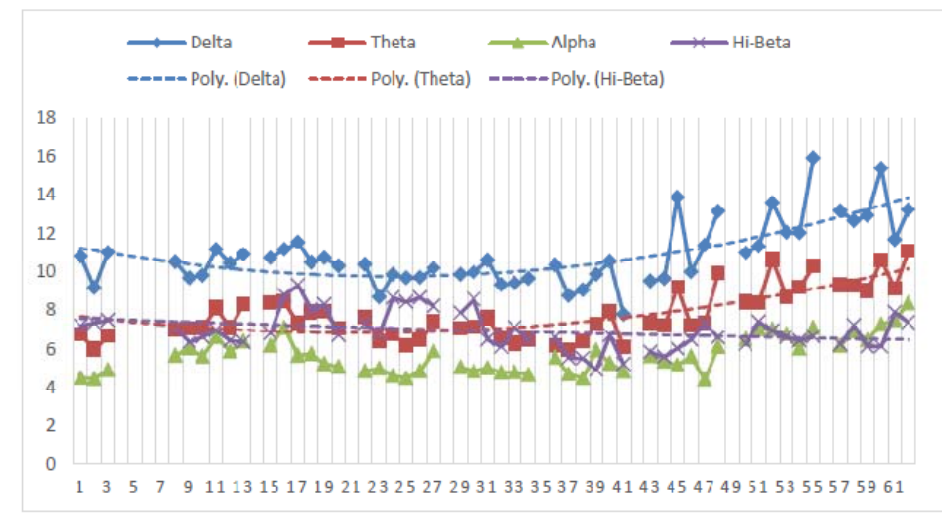

The graph above shows the session on 19th May 2015

ii. Session three: Gut directed hypnotherapy and self-hypnosis rehearsal

- The first 5 period, client has learned how to experience the tense as well as calm feeling. Thus, tension associated Hi-Beta was increasing during first 5 period.

- After 5 period, the tension associated Hi-Beta brainwaves start to decrease. Alpha decreased could indicate that his brain stop thinking too much and getting calmer. 
South American Journal of Academic Research

Special Edition May 2016

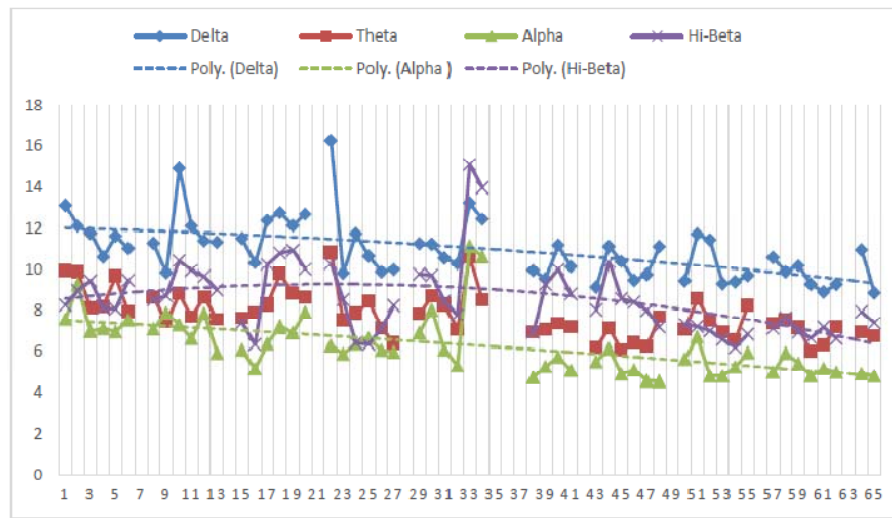

The graph above shows the session on 25th May 2015

\section{Remark}

Beta brainwaves are associated with cortical cell activity, active and working; fast brainwaves.

Hi Beta brainwaves are associated with stress, anxiety and tension.

Alpha brainwaves are associated with relaxed focus, light trance, calm, intuition, beginning access to subconscious mind, increased serotonin production, not thinking.

Theta brainwaves are associated with dreaming, drowsy and subconscious mental activities

Delta brainwaves are associated with physical healing, sleep, unconscious mind and deep relaxation; slowest brainwaves.

\subsection{Therapeutic outcome}

Eric Liu reports that he feels a significant improvement after 3 sessions. He reports that although he didn't practice coping skill much, he is still able to relax by practicing the personalized self-hypnosis alone. Now he feels no more worry about worst things will be happened on him and not afraid of his IBS issues. He feels that his stomach discomfort problem becomes mildly and the frequency reduce to the minimum already. The nervous feelings have also significantly reduced after first and second therapy sessions.

Now, he is able to control and even stop himself to go into the washroom repeatedly before he goes out from the house. He found that personalized self-hypnosis method is impressive because he can control the pain and discomfort just by putting his palm over his stomach. He feels that his overall life quality has improved since the first session.

After 1 month follow up, he remarks that he didn't experience any IBS related issues at all. No more Stomach and liver area discomfort.

\subsection{Further recommendations}

Following the therapy session the most appropriate management of his issues:

i. Nutritional Therapy. This will enable him to stabilize his mood and anxious issues.

ii. Emotional Freedom Techniques. This coping technique can help him to overcome all nervousness and stress related issues.

iii. Personalized self-hypnosis. He should keep practice his personalized self-hypnosis technique to improve his brainwaves and IBS issues.

iv. Counselling session/psychotherapy/hypnotherapy for stress management and mood stabilization.

v. Progressive muscle relaxation therapy 
South American Journal of Academic Research Special Edition May 2016

\section{Reference}

[1.] Perjohan Lindfors, Peter Unge, Patrik Arvidsson, Henry Nyhlin, Einar Björnsson, Hasse Abrahamsson, Magnus Simrén (2012). Effects of Gut-Directed Hypnotherapy on IBS in Different Clinical Settings-

Results From Two Randomized, Controlled Trials Journal: American Journal of Gastroenterology 2012. doi: 10.1038/ajg.2011.340 